\title{
INTERVENTION FOR BURNOUT AMONG INSTITUTE OF AFRICAN STUDIES POSTGRADUATE STUDENTS: IMPLICATIONS FOR INSTITUTE OF AFRICAN STUDIES PERSONNEL
}

\author{
IBENEKWU, IKPECHUKWUKA E ${ }^{1}$, OKAGU, GEORGE OGBONNA ${ }^{2 *}$, IWUNDU, I.E ${ }^{3}$, MATHEW, \\ IKECHUKWU EZE ${ }^{4}$, JOHN CHIDUME NWAOGAIDU ${ }^{5}$, OGIRI, HAPPINESS KODICHINMA ${ }^{6}$ \& \\ NWANGWU, TOCHUKWU $\mathbf{L}^{7^{*}}$ \\ 1,2,3,4,5,6 Institute of African Studies, University of Nigeria, Nsukka
}

${ }^{7}$ Department of Educational Foundations, University of Nigeria, Nsukka

\begin{abstract}
The purpose of this research was to examine the Effect of Rational Emotive Behaviour Therapy (REBT) on Burnout among Institute of African Studies Postgraduate Students in South-Eastern Nigeria Universities.

Method: The research adopted a randomised controlled group design. 68Institute of African Studies post-graduate students with high burnout symptom level in the participating universities took part in the study. They were assigned randomly to groups - intervention $(n=34)$ and control groups $(n=34)$. Data was collected using Oldenburge Burnout Inventory for students (OLBI-S) and analysed using $2 \times 2$ ANOVA at $P<.05$.

Result: From the findings, result showed that level of burnout among Institute of African Studies postgraduate students in the intervention group significantly reduced as a result of the REBT program in-relation to those of no-intervention group as rated by the OLBI-S subscales. Also, beneficiaries of the REBT program retained minimal level of burnout 14 days prior to follow-up.

Conclusion: From the present finding, the REBT program is a helpful intervention for preventing and managing burnout among postgraduate students hence, the REBT ideology and practice is required in the school system by the Institute of African Studies personnel for effective students' all round improvement.

KEYWORDS: Intervention, Burnout, Postgraduates, Institute of African Studies, Personnel
\end{abstract}

Received: Jun 10, 2020; Accepted: Jun 30, 2020; Published: Jul 27, 2020; Paper Id.: IJMPERDJUN2020478

\section{INTRODUCTION}

The learning environment is a studious and strenuous one considering a whole lot of activities involved. Students encounter a lot of stress ranging from home expectations, personal aspirations and meeting other academic goals in school $^{1}$. Higher education is an arduous task in which students work with the aim of attaining a degree certificate through attending lectures, making-up notes, studying to pass courses and other relationship matters in school ${ }^{1,2}$. This is believed to affect their leisure time, thereby, worsening stress among students ${ }^{3,4}$. As a result, burnout gradually develops throughout the academic years ${ }^{5,6,7}$. As a result of academic activities in school students devout much time on academic task. As such, students' ability to study and engage in academic activities would be hindered coupled with inadequate school climate, incessant strikes, conflicts between students-students and students-lecturers. Such scenario could trigger fatigue, emotional and mental weariness (burnout) ${ }^{8}$.Burnoutis defined as a syndrome of emotional tiredness that is an aftermath of high seeming academic task, the growth of a 
detached approach to one's studies and feelings of inadequacy as a higher education student $9 ; 2$. These stressors can be academic activities ${ }^{10,13}$ Recently, research focus has moved from burnout to academic activities in the school ${ }^{11}$. Academic activities is demanding right from undergraduate through postgraduate studies, especially among students whose research focus is on all aspects of life and the culture of Africans. Hence, Institute of African Studies'postgraduate students seem to be prone to burnout due to stresses emanating from strenuous academic activities.

Due to the importance of Institute of African Studies program, identifying and assisting Institute of African Studies postgraduate students experiencing burnout is worthwhile because of the relevance of this group of students in acheiving the objectives ofInstitute of African Studies. In Nigeria, the vision ofInstitute of African Studies programme is to operate as a research and post-graduate teaching center with emphasis on all aspects of life and the culture of Africans. Specifically, Institute of African Studies aims at promoting a serious study of Nigerian societies and cultures with particular emphasis on the peoples of the south-eastern states; providing research opportunities and experience to young Nigerian scholars and others; providing an institutional base for visiting Africans from other universities; creating and sustaining an academic environment in which scholarly enterprises can flourish and interdisciplinary efforts promoted and regarded; building up institutional resources such as a research library, museum, art gallery, cinema studio and visual library et cetera, in support of the University's research and teaching functions and supporting a publications programme that gives visibility to the works of the Institute and also other activities sponsored by it ${ }^{12}$. For these objectives to be fully attained, Institute of African Studies'postgraduates needs to be mentally, emotinally and physically fit to tackle varying academic tasks. Furthermore, it seems these students suffer from burnout issues without help. This implies that personnel who handle Institute of African Studies' postgraduates require adequate skills inorder to effectively manage diverse students' problems. Though from the extant literature, it suggests that so much research and studies have been carried out on students burnout, the evidence in a Nigerian context is thin particularly for Institute of African Studiespostgraduates. However, evidence has shown the effectiveness of REBT in containing burnout among students ${ }^{12}$. This study, therefore, sought to identify the effect of Rational Emotive Behaviour Therapy (REBT) on burnout among Institute of African Studies'postgraduates. To achieve this purpose, a research question guided the study (a) what is the effect of a psychoeducational programme embedded in the theory of rational-emotive behavior therapy in reducing burnout among Institute of African Studies'postgraduates?

\section{THEORETICAL REVIEW}

Albert Ellis (1955) theory of rational-emotive behavior therapy posits the adoption of a cognitive behavioral approach in reducing burnout.

\section{METHOD}

The study met the ethical conditions and was conducted in accordance with the canons of Declaration of Helsinki. All Institute of African Studiespostgraduate students who were available at the time of the study was conducted were eligible to participate.Consent for participation was obtained from all participants. Experimental research design was used. This study comprised of 68 Institute of African Studiespostgratuate students in public institutions in southeastern Nigeria who met the inclusion criteria of beingInstitute of African Studiespostgraduate students, not involved in any other burnout intervention program during the time of the study. G-power 3.1 software ${ }^{13}$ was used to determine the sample size with $\mathrm{P}<$ 0.05 and statistical power of 0.89 for repeated measures between -factors F-test analysis of variance (ANOVA). The instrument for data collection was Oldenburg Burnout Inventory for students (OLBI-S)-a well known burnout assessment 
instrument adapted and validated with 16 questions designed to measure academic burnout of students. Each of the subscale have eight questions modelled on a 4-point rating scale of strongly agree, agree, disagree and strongly disagree ${ }^{10}$. To determine burnout, the negatively worded items are reversed before the scores are summed up so that high scores determines high level of exhaustion and disengagement. Participants who scored high (total burnout score of 49 and above) on the OLBI-S during the pre-test were involved in this study. Questions related to basic demographics of the participants was sought such as age,gender, year of study, residence (campus or off-campus) and parental status. The two dimensions measured exhaustion and disengagement with Cronbach's alpha value of 0.86 and 0.88 respectively. Thus, the instruments were reliable.

\section{PROCEDURE}

Data collected for the study was done by the researchers between November 2019 and February 2020 from two participant groups (intervention and no-intervention control groups), with three measures (pretest, posttest, and follow-up test) administered. The students were then randomly assigned to either of the two groups- intervention group $(n=34)$ and control group $(\mathrm{n}=34)$ using Random Allocation Software ${ }^{14}$. The procedure adopted for assigning students to the groups was as described in Onuigbo et al ${ }^{15}$. To minimize bias, sealed, opaque envelopes were used to conceal the allocation sequence from the data analysts.

\section{INTERVENTION}

\section{REBT Burnout Treatment Manual}

The burnout treatment program was conducted for 12 weeks with 2 sessions in a week lasting for 60 minutes. In each of the sessions, the treatment involves guiding the postgraduate students to identify and note their school and non-school related stressors, irrational beliefs and negative thoughts that begets burnout.

\section{METHOD OF DATA ANALYSIS}

All data collected were analyzed using SPSS Version 22. Repeated measures analysis of variance (ANOVA) was used to determine the main effect of treatment condition, the main effect of time and time $\times$ group interaction effect. Partial eta squared was calculated to show the effect size of the REBT treatment intervention. Since the group effect, time effect and the time $\times$ group interaction effect were all significant, a post-hoc analysis was performed with the bonferroni correction in order to determine the specific significant effect of the intervention for the different times for each of the group. The result showed that sphericity assumption was met for Exhaustion subscale $\left(\chi^{2}(2)=3.081 ; p=0.214\right)$ but not met for Disengagement $\left(\chi^{2}(2)=9.679 ; P=0.008\right)$.

\section{RESULTS}

The mean age of both the intervention and control groups was shown to be significant at $21.06 \pm 1.83$ and $20.38 \pm 1.41$ respectively. We had more females both in the study (62\%)with majority living in campus (53\%). The study was conducted in the southeastern part of Nigeria, majorly Igbo ethnic group.

The result of the two-way mixed ANOVA indicated an overall significant main effect of time on students' burnout level for each of the burnout subscales as recorded by the OLBI-S [Exhaustion: $\mathrm{F}(2,170)=115.16, P<0.001, \eta_{p}{ }^{2}=$ 0.575; Disengagement: $\left.\mathrm{F}(2,158.28)=451.17, P<0.001, \eta_{p}{ }^{2}=0.841\right]$. With an average effect on students' exhaustion and a very high effect on students' disengagement as indicated by the values of the partial eta squared, the REBT program had an 
overall high significant effect over time on postgraduate students' burnout levels. Similarly, there was a significant main effect of group on postgraduate students' burnout level as shown in the OLBI-S subscales [Exhaustion: $F(1,85)=192.94$, $P<0.001, \eta_{p}{ }^{2}=0.694$; Disengagement: $\left.\mathrm{F}(1,85)=686.02, P<0.001, \eta_{p}{ }^{2}=0.890\right]$. From this we can deduce that the REBT group which participants belonged had an overall high significant effect on their burnout level as indicated by the value of partial eta squared which represent the effect size of the REBT program. Thus, overall, students who benefited from the REBT program (intervention group) had a significantly different (lower) burnout scores when compared to those who did not benefit from the program.

ANOVA result indicated that the interaction between Time and Group was significant as shown in the OLBI subscales [Exhaustion: $\mathrm{F}(2,170)=128.13, P<0.001, \eta_{p}{ }^{2}=0.601$; Disengagement: $\mathrm{F}(1.86,155.28)=437.92, P<0.001, \eta_{p}{ }^{2}=$ 0.837]. This means that depending on participant's group, his/her burnout level would change over time. To further validate these observations and ascertain the exact direction and location of the significant differences and change in burnout levels by time, group and time*group interactions, we conducted a post-hoc analysis for pair wise comparison using Bonferroni.

There was a significant difference in mean scores in both the engagement and exhaustion subscales of the OLBI for Time 2 and Time 3 in the two groups (intervention and control) with all p-values $<0.001$. The results revealed a steady significant decline in the burnout scores of the students in the intervention group over time (particularly in Time 2 and follow-up) when compared to students in the control group. This significant change in burnout scores is linked to the REBT program. Thus, these outcomes indicated the efficacy of the REBT program in containing burnout among Institute of African Studies' postgraduates.

\section{DISCUSSION}

The results of the study showed the effectivesness of REBT intervention on burnout among Institute of African Studiespostgraduate students who received the treatment and participated in the REBT programin contrast to students in the control group who did not participate in the intervention program. However before the pretest assessment, high levels of burnout were found among the participants. There was a significant decline among Institute of African Studiespostgraduate students in the intervention control group, their burnout level significantly reduced when compared to those of the students in the control group who did not participate in the intervention program.This finding is in line with previous studies, which indicated that REBT was an effective approach for reducing burnout ${ }^{16,17,18,19}$ A study showed that REBT intervention resulted in a significant reduction in burnout among Institute of African Studiespostgraduate students ${ }^{20}$. The REBT treatment group showed a continuous reduction from post-test to follow-up. This showed a positive effect of REBT on reduction of burnout when applied as an intervention for such, especially among Institute of African Studiespostgraduate students. These findings align with previous studies which revealed the therapeutic helpfulness of REBT in reducing burnout ${ }^{21}$.

\section{CONCLUSIONS}

This study examined the effect of Rational Emotive Behaviour Therapy (REBT) as a burnout management intervention among Institute of African Studiespostgraduate students in southeastern Nigeria. At posttest and follow-up, the result showed a significant effect of REBT in reducing the symptoms of student burnout in the intervention group compared to those of the control group as recorded in the OLBI-S subscales. Therefore, we recommended that since the REBT program 
was helpful at reducing burnout symptoms among postgraduate students, the Institute of African Studies personnel should imbibe the REBT practice in teaching the Institute of African Studies postgradute students for effective promotion of students' overall well-being and development.

\section{REFERENCES}

1. Robotham, 2008 D. Robotham Stress among higher education students: Towards a research agenda Higher Education, 56 (2008), pp. 735-746.

2. Schaufeli, I. Martinez, A.M. Pinto, M. Salanova, A. Bakker Burnout and engagement in university students: A cross-national study. Journal of Cross-Cultural Psychology, 33 (5) (2002), pp. $464-481$.

3. Tarnowski, M., and Carlotto, M.S.(2007) Burnout syndrome in students of psychology. TemasemPsicologia, 15(2):173-180.

4. Aguiar, S.M., Vieira, APGF., Vieira, KMF, No 'b JO, Aguiar SM. Prevalência de sintomas de estresseemestudantes de medicina. J Bras Psiquiatr. 2009;58(1):34-38. doi: 10.1590/S0047-20852009000100005.

5. Fares, J., Saadeddin, Z., Al Tabosh, H., Aridi, H., El Mouhayyar, C., Koleilat, M.K, Chaaya, M., and El Asmar, K. (2016)..Extracurricular activities associated with stress and burnout in preclinical medical students. Journal of epidemiology and global health, 6(3):177-185. doi: 10.1016/j.jegh.2015.10.003.

6. Pagnin, D., and Queiroz, V.(2015). Influence of burnout and sleep difficulties on the quality of life among medical students. Springerplus, 4(1):676. doi: 10.1186/s40064-015-1477-6.

7. Chang, E., Eddins-Folensbee, F., and Coverdale, J. (2012).Survey of the prevalence of burnout, stress, depression, and the use of supports by medical students at one school. Acad Psychiatry, 36(3):177-182. doi: 10.1176/appi.ap.11040079.

8. Schaufeli, W.B. and Greenglass, E.R. (2001) Introduction to Special Issue on Burnout and Health. Psychology and Health, 16, 501-510. http://dx.doi.org/10.1080/08870440108405523

9. Salmela-Aro, Kiuru et al., 2009. Achievement strategies during university studies predict early career burnout and engagement. Journal of Vocational Behavior, 75 (2) (2009), pp. 162-172.

10. Maslach, C. et al. (1996). Maslach Burnout Inventory - General Survey In: Maslach C, editor; , Jackson SE, editor; , Leiter MP, editor. (eds). Maslach Burnout Inventory manual, 3rd ed. Palo Alto: Consulting Psychologists Press, 19-26.

11. Fredricks, P.C. Blumenfeld, A.H. Paris School engagement: Potential of the concept, state of the evidence. Review of Educational Research, 74 (2004), pp. 59-109. CrossRefView Record in ScopusGoogle Scholar

12. https://www.unn.edu.ng/institute-of-african-studies-history/

13. Faul, F., Erdfelder, E., and Buchner, A., et al. (2009).Statistical Power Analyses Using G* Power3.1: Tests for Correlation and Regression Analyses. Behav Res Methods 2009;41:1149-60.

14. Saghaei, M. Random Allocation Software. $2004 . \quad$ Available At: Http://Mahmoodsaghaei.Tripod.Com/Softwares/Randalloc.Html. [Accessed October 18, 2018].

15. Onuigbo, L.N, Eseadi, C., Ugwuoke, S.C, et al.(2018). Effect of rational emotive behavior therapy on stress management and irrational beliefs of special education teachers in Nigerian elementary schools. Medicine (Baltimore), 97(37): e12191.

16. Ogbuanya TC, Eseadi C, Orji TC, et al. Effects of rational emotive coaching on occupational stress and work ability among electronics workshop instructors in Nigeria. Medicine (Baltimore) 2017;96:e6891.

17. Lupu V, Ifene F. The impact of rational emotive behavior education on anxiety in teenagers. J Cogn Behav Psychother 2009;9:95-105. 
18. Szentagotai A, Freeman A. An analysis of the relationship between irrational beliefs and automatic thoughts in predicting distress. J Cogn Behav Psychother 2007;7:1-9.

19. Trip S, Vernon A, Mcmahon J. Effectiveness of rational-emotive education: a quantitative meta-analytical study. J Cogn Behav Psychother 2007;7:81-93.

20. Jesus SN, Conboy J. A stress management course to prevent teacher distress. IJEM 2001;15:131-7. [Google Scholar]

21. Ogbuanya TC, Eseadi C, Orji CT, et al. Effect of rational-emotive behavior therapy program on the symptoms of burnout syndrome among undergraduate electronics work students in Nigeria. Psychol Reports 2018;33294117748587.

22. Jaseena, Fathima, and Salih Moosa. "The Philosophy of Data Literacy-An Analysis among Higher Education Learners." International Journal of Educational Science and Research (IJESR) 10.1 (2020):7-14.

23. Siddiqui, Kalim. "Higher Education in the Era of Globalization." International Journal of Humanities and Social Sciences (IJHSS) 3.2 (2014): 9-32.

24. Tsikati, Alfred F., Marietta P. Dlamini, and Musa A. Dube. "Research Methodologies Used by Postgraduate Agricultural Education in Eswatini." International Journal of Agricultural Science and Research (IJASR) 9.2 (2019):43-52.

25. Gautam, Diksha, and Deepa Vinay. "Development \& standardization of a scale to measure smartphone addiction among college students." International Journal of Educational Science and Research (IJESR) 8.2 (2018): 87-94. 